\title{
Biodiscovery of Potential Antibacterial Diagnostic Metabolites from the Endolichenic Fungus Xylaria venustula Using LC-MS-Based Metabolomics
}

\author{
Krystle Angelique A. Santiago ${ }^{1,2}$, RuAngelie Edrada-Ebel ${ }^{3}$, Thomas Edison E. dela Cruz ${ }^{4}$, Yuen Lin Cheow ${ }^{1,2}$ \\ and Adeline Su Yien Ting ${ }^{1,2, * \mathbb{D}}$
}

1 School of Science, Monash University Malaysia, Jalan Lagoon Selatan, Bandar Sunway, Selangor Dahrul Ehsan 47500, Malaysia; krystle.santiago@monash.edu (K.A.A.S.); cheow.yuen.lin@monash.edu (Y.L.C.)

2 Tropical Medicine \& Biology Multidisciplinary Platform, Monash University Malaysia, Jalan Lagoon Selatan, Bandar Sunway, Selangor Dahrul Ehsan 47500, Malaysia

3 Strathclyde Institute of Pharmacy and Biomedical Sciences, University of Strathclyde, The John Arbuthnott Building, 161 Cathedral Street, Glasgow G4 0RE, UK; ruangelie.edrada-ebel@strath.ac.uk

4 Department of Biological Sciences, College of Science, University of Santo Tomas, España Boulevard, Manila 1008, Philippines; tedelacruz@ust.edu.ph

* Correspondence: adeline.ting@monash.edu

check for updates

Citation: Santiago, K.A.A.; Edrada-Ebel, R.; dela Cruz, T.E.E.; Cheow, Y.L.; Ting, A.S.Y. Biodiscovery of Potential Antibacterial Diagnostic Metabolites from the Endolichenic Fungus Xylaria venustula Using LC-MS-Based Metabolomics. Biology 2021, 10, 191. https://doi.org/ 10.3390/biology10030191

Academic Editor: Gill Diamond

Received: 31 December 2020

Accepted: 16 February 2021

Published: 4 March 2021

Publisher's Note: MDPI stays neutral with regard to jurisdictional claims in published maps and institutional affiliations.

Copyright: (c) 2021 by the authors. Licensee MDPI, Basel, Switzerland. This article is an open access article distributed under the terms and conditions of the Creative Commons Attribution (CC BY) license (https:// creativecommons.org/licenses/by/ $4.0 /)$.
Simple Summary: In this study, we determined the bioactivities and chemical natures of three species of lichen Usnea and their associated endolichenic fungi (ELF) through metabolomics. We found significant differences in the antibacterial activities and the metabolites produced by the host lichen and its ELF, with the latter targeting a wider scope of organisms. We also discovered potential key metabolites produced by ELF that are yet to be reported. This study shows the application of metabolomics in rapidly identifying bioactive metabolites that are of significance in the discovery of new drugs.

Abstract: Three species of the lichen Usnea (U. baileyi (Stirt.) Zahlbr., U. bismolliuscula Zahlbr. and $U$. pectinata Stirt.) and nine associated endolichenic fungi (ELF) were evaluated using a metabolomics approach. All investigated lichen crude extracts afforded antibacterial activity against Staphylococcus aureus (minimum inhibitory concentration (MIC): $0.0625 \mathrm{mg} / \mathrm{mL}$ ), but none was observed against Escherichia coli, while the ELF extract Xylaria venustula was found to be the most active against S. aureus (MIC: $2.5 \mathrm{mg} / \mathrm{mL}$ ) and E. coli (MIC: $5 \mathrm{mg} / \mathrm{mL}$ ). X. venustula was fractionated and tested for to determine its antibacterial activity. Fractions XvFr1 to 5 displayed bioactivities against both test bacteria. Selected crude extracts and fractions were subjected to metabolomics analyses using high-resolution LC-MS. Multivariate analyses showed the presence of five secondary metabolites unique to bioactive fractions $\mathrm{XvFr}_{\mathrm{v}}$ to 3 , which were identified as responsible for the antibacterial activity of $X$. venustula. The $p$-values of these metabolites were at the margin of significance level, with methyl xylariate $C\left(P \_60\right)$ being the most significant. However, their high variable importance of projection (VIP) scores $(>5)$ suggest these metabolites are potential diagnostic metabolites for $X$. venustula for "dual" bioactivity against $S$. aureus and E. coli. The statistical models also showed the distinctiveness of metabolites produced by lichens and ELF, thus supporting our hypotheses of ELF functionality similar to plant endophytes.

Keywords: dereplication; endolichenic; LC-MS; metabolomics; OPLS-DA; PCA; PLS-DA; Xylaria

\section{Introduction}

As symbiotic organisms, lichens are known as unique sources of bioactive secondary metabolites called lichen substances [1]. The mycobiont, which is the fungal component 
of the lichen, is reported to produce approximately 1000 chemically diverse lichen substances [2]. Most of these metabolites are unique to lichens, with only a small portion occurring in fungi and higher plants [3]. These substances were found to exhibit significant biological activities including antibacterial [4], antifungal [5], anti-inflammatory [6], antioxidant [7], antiviral [8] and cytotoxic [9] activities. Nevertheless, their slow growth in nature limits the widespread application of these substances, particularly for pharmaceutical uses [10]. In addition, overcollection of lichens impacts their conservation. Hence, other fast-growing and easily cultured organisms, in contrast to slow-growing lichens, are prioritized. Interestingly, a group of asymptomatic fungi that resemble plant endophytes was discovered thriving inside healthy lichen thalli $[11,12]$. This group of fungi, known as endolichenic fungi (ELF), was first described in 1990 [13]. In our other study, we reported very diverse species of ELF from Usnea which differed significantly between geographic origins-e.g., Philippines vs. Malaysia $[14,15]$. Since then, over 35 species of ELF have been isolated and characterized [16]. Furthermore, ELF are also known to possess secondary metabolites that are hypothesized to be distinct from those produced by lichens [17]. Few studies, however, have been conducted to specifically view such distinction. Since the first report on the metabolites produced by ELF in 2007 [18], a tremendous increase in the number of compounds has been described. To date, 351 secondary metabolites produced by ELF have been characterized and identified, 196 of which were novel [16]. These metabolites consisted of a diverse array of structural types that included alkaloids, quinones, oxygenated heterocycles, aromatic compounds, terpenes, peptides, steroids, and allylic compounds, which were also known to exhibit various biological activities [19-23]. ELF have therefore offered another avenue for drug discovery.

The search for new bioactive natural products for drug discovery has always been a challenge. Metabolomics, a comprehensive profiling tool used to study and quantify small molecules present in a biological system [24], is gaining popularity in speeding up the discovery of active and novel drugs. It is considered as the most recent member of the "omics" technology, which involves advanced instrumentation, analytical, and statistical methodologies and well-established metabolic pathways and large metabolite databases [25]. An integral component of metabolomics, called dereplication, is the recognition and elimination of already known substances in the early stages of the screening process in a drug discovery scheme [26]. Through dereplication, the discovery of bioactive and novel natural products is accelerated [27].

In this study, a metabolomics approach was adopted to identify potentially novel and active secondary metabolites from the three species of Usnea and their associated ELF. Using the generated LC-MS data, statistical multivariate data analyses, i.e., principal component analysis (PCA), partial least squares discriminant analysis (PLS-DA) and orthogonal projection to latent structures discriminant analysis (OPLS-DA), were carried out to capture the significant metabolites produced uniquely by the host lichen Usnea and its associated ELF with antibacterial activities against Staphylococcus aureus. The obtained data of this study were analyzed and integrated to prioritize the specific lichen and ELF producing interesting compounds for future isolation work. Finally, this study also aimed to compare the chemical profiles and antibacterial properties of lichens and ELF crude extracts, which may provide an interesting insight on the possible role of ELF in their lichen hosts.

\section{Materials and Methods}

\subsection{The Test Organisms: The Lichen Usnea and the Isolated Endolichenic Fungi (ELF)}

Three lichens, $U$. baileyi (Stirt.) Zahlbr., $U$. bismolliuscula Zahlbr., and $U$. pectinata Stirt., were collected from barks of pine trees (Pinus merkusii) growing dominantly in Bukit Larut in Perak, Malaysia $\left(4^{\circ} 51^{\prime} 44^{\prime \prime} \mathrm{N}, 100^{\circ} 47^{\prime} 36^{\prime \prime}\right.$ E) and Sagada in Mountain Province, Philippines $\left(16^{\circ} 5^{\prime} 30^{\prime \prime} \mathrm{N}, 120^{\circ} 50^{\prime} 48^{\prime \prime} \mathrm{E}\right)$. This pine tree species is native to the Philippines but was introduced in Peninsular Malaysia in the 1950s for reforestation [28]. These trees, approximately $>30$ years old, were found in nonplantation areas. Permission to collect lichens was obtained from nearby land owners. Collection of lichens on the tree trunk was 
carried out one meter from the ground and only those lichens with lengths of $>1.5$ inches were collected. Extra care was taken to ensure that no trees were wounded or injured during the collection. These lichen specimens were placed in sterile plastic bags and properly labeled with location, elevation, and global positioning system (GPS) data. These lichens were processed within 24 to $48 \mathrm{~h}$. From these lichens, ELF were isolated following the protocol of Samanthi et al. [21] with modifications of the ethanol concentration and an additional step of plating the rinsed water onto Potato Dextrose Agar (PDA; Merck, Darmstadt, Germany) to validate the efficacy of the surface sterilization. Briefly, the lichen thalli were surface-sterilized by successive dipping on various ethanol concentrations $(70 \%$, $80 \%, 85 \%$, and $90 \% \mathrm{v} / \mathrm{v}$ ) for $1 \mathrm{~min}$, and then in $10 \%$ sodium hypochlorite ( $\mathrm{NaOCl}$ ) for $30 \mathrm{~s}$ and $95 \%$ ethanol for $30 \mathrm{~s}$. The surface-sterilized lichen thalli were cut into small fragments and plated onto $2 \%$ malt yeast extract agar (MYE, Lab M Limited, Heywood, UK). The ELF isolates were then identified by sequence analysis of the ITS region of the rRNA and deposited on GenBank under specific accession numbers. Specific details on the isolation, identification, and diversity assessment of the lichen hosts and the associated ELF have been separately reported in our other studies [14,15].

\subsection{Extraction of Secondary Metabolites Produced by Lichen Usnea and ELF}

Six lichen specimens, three representing each study site, and nine ELF isolates, were extracted for their secondary metabolites (Table 1). These ELF isolates were selected based on their abundance, unique morphologies, and relatively short incubation periods (i.e., $<7$ days). The extraction of lichen acids was carried out following the protocol of Santiago et al. [29]. Briefly, one gram of each lichen sample was ground until powdery then macerated with $10 \mathrm{~mL}$ of acetone overnight. The crude extract was filtered using a sterile filter paper (Whatman no. 1, Tisch Scientific, North Bend, OH, USA) and the supernatant was collected in a preweighed vial. Each vial was carefully covered with a sterile cheese cloth until the acetone evaporated completely to yield the crude extract. For the extraction of the ELF secondary metabolites, a solid-state fermentation was carried out. Each ELF isolate was initially grown on PDA for 15 days under light at $26 \pm 2{ }^{\circ} \mathrm{C}$ for $12 \mathrm{~h}$. After incubation, 10 fungal agar blocks ( $15 \mathrm{~mm}$ in diameter) were inoculated in $500 \mathrm{~mL}$ Erlenmeyer flasks containing $200 \mathrm{~mL}$ of Glucose Malt Yeast Extract (GMY; Lab M Limited, Lancashire, UK) $(9 \times 200 \mathrm{~mL})$ broth and incubated at $26 \pm 2{ }^{\circ} \mathrm{C}$ on a rotary shaker $(135 \mathrm{rpm})$ for 15 days at room temperature $\left(26 \pm 2{ }^{\circ} \mathrm{C}\right)$ to obtain the seed culture. Scale-up fermentation was carried out for all nine ELF strains in five $1 \mathrm{~L}$ Erlenmeyer flasks (five replicates), each containing 160 grams of rice and $240 \mathrm{~mL}$ of distilled water [30]. The rice media were then autoclaved, and each flask was inoculated with $25 \mathrm{ml}$ of the seed culture and incubated at $26 \pm 2{ }^{\circ} \mathrm{C}$ for 50 days.

\subsection{Antibacterial Activities of the Lichen and ELF Crude Extracts}

Paper disk diffusion and agar well assays (File S1) were used to evaluate the antibacterial properties of lichen and ELF crude extracts, respectively, following Clinical and Laboratory Standards Institute (CLSI) recommendations [31]. The test organisms used were Escherichia coli ATCC 25922 and Staphylococcus aureus ATCC 25923. The antibacterial activities of the crude extracts presented in this paper have also been reported in our recent works $[14,15]$. However, the inclusion of these data in the current metabolomic analyses is necessary to compare the bioactivities of the crude extracts with those of their respective fractions. In addition, the minimum inhibitory concentrations (MICs) and minimum bactericidal concentrations (MBCs) of all crude extracts were also determined (File S1). The MIC and MBC values presented in this study were reported in our earlier works [14,15]. These data are again presented here to illustrate the selection criteria for the succeeding fractionation works. 
Table 1. The nine endolichenic fungi (ELF) species isolated from Usnea lichens collected from Bukit Larut, Malaysia (MY), and Sagada, Philippines (PH).

\begin{tabular}{|c|c|c|c|c|}
\hline ELF Species & $\begin{array}{c}\text { GenBank } \\
\text { Accession Number }\end{array}$ & Lichen Host & Criterion for Selection & Reference $^{1}$ \\
\hline Xylaria sp. & MN071376 & U. bismolliuscula & Abundance & \multirow{4}{*}{ Santiago et al. [15] } \\
\hline $\begin{array}{l}\text { Fusarium solani } \\
\text { (Mart.) Sacc. }\end{array}$ & MG881825 & U. pectinata & \multirow{3}{*}{ Unique morphology } & \\
\hline $\begin{array}{l}\text { Pseudopestalotiopsis theae } \\
\text { (Sawada) Maharachch., } \\
\text { K.D. Hyde and Crous }\end{array}$ & MG881833 & U. pectinata & & \\
\hline Xylariaceae sp. & MN071378 & U. pectinata & & \\
\hline $\begin{array}{l}\text { Astrocystis bambusae } \\
\text { (Henn.) Lćssře et Spooner }\end{array}$ & MH370741 & U. bismolliuscula & Unique morphology & \multirow{5}{*}{ Santiago et al. [14] } \\
\hline $\begin{array}{l}\text { Annulohypoxylon albidiscum } \\
\text { J.F. Zhang, J.K. Liu, K.D. } \\
\text { Hyde and Z.Y. Liu }\end{array}$ & MH370738 & U. pectinata & \multirow{2}{*}{$\begin{array}{l}\text { Short incubation period } \\
\text { (fast growth) }\end{array}$} & \\
\hline $\begin{array}{l}\text { Daldinia eschscholtzii } \\
\text { (Ehrenb.) Rehm }\end{array}$ & MN071367 & U. pectinata & & \\
\hline $\begin{array}{l}\text { Nemania bipapillata (Berk. } \\
\text { and M.A. Curtis) Pouzar }\end{array}$ & MN071354 & \multirow[t]{2}{*}{ U. baileyi } & Abundance & \\
\hline Xylaria venustula Sacc. & MH370742 & & Unique morphology & \\
\hline
\end{tabular}

${ }^{1}$ Specific details on the collection sites and isolation and identification of these ELF species were separately reported in our other studies.

\subsection{Selection of Lichen and ELF Crude Extracts for Fractionation Work}

Two criteria for selecting lichen and ELF crude extracts were considered in this study: (1) high metabolic diversity and (2) moderate to strong antibacterial activities. For the assessment of the metabolic diversity, analysis of the mass spectra of the crude extracts was carried out using MetaboAnalyst@ 4.0 [32]. Key features of this process, which are incorporated in the software, include data upload and cleaning, processing, normalization, statistical analyses, and annotation. Heatmaps, together with hierarchical clustering techniques, were generated using the software's default settings. For the antibacterial activities, extracts with zones of inhibition (ZOIs) of $\geq 10 \mathrm{~mm}$ and low MIC and MBC values $(\leq 10 \mathrm{mg} / \mathrm{mL}$ ) were considered. Selection of crude extracts to be fractionated resulted in a small sample size of this study. As such, possible biases during analyses may be present. Nevertheless, the data presented in this study may provide preliminary information regarding the metabolomics analyses of lichen and ELF crude extracts.

\subsection{Fractionation and Initial Characterization of Selected Lichen and ELF Crude Extracts}

Lichen and ELF crude extracts that exhibited moderate to strong antibacterial activities ( $\geq 10 \mathrm{~mm}$ ZOI) were selected for fractionation using silica gel column chromatography. Thin layer chromatography (TLC) was first carried out with aluminum precoated silica gel $60 \mathrm{~F}_{254}$ plates (Merck, Darmstadt, Germany). Spots were then visualized under UV light $(\lambda=254 \mathrm{~nm})$. Following TLC, silica gel chromatography (silica gel 60, Merck, Darmstadt, Germany) was performed. An isocratic system of toluene/acetic acid (85:15) was used as the mobile phase for lichen crude extracts. The ELF crude extracts were fractionated using a gradient of dichloromethane and methanol (DCM/MeOH, 90:10, 80:20, 70:30, 60:40 and 50:50), with solvent volume ranging between 200 and $1000 \mathrm{~mL}$ until the collected fraction was rendered colorless. All solvents used were of analytical grade. Following fractionation, the lichen and ELF crude extracts and fractions, including usnic acid and the solvents acetone and ethyl acetate, were subjected to high-resolution LC-MS (LC-HRMS) analysis. LC-MS was carried out using an Agilent 1290 Infinity LC system coupled to an Agilent 6520 Accurate-Mass-Q-TOF mass spectrometer with dual ESI source instrumentation 
(California, USA) equipped with Agilent Zorbax Eclipse XDB-C18 (Santa Clara, CA, USA). A $2.1 \times 150 \mathrm{~mm}$ column was used with the following parameters: column temperature at $25^{\circ} \mathrm{C}, 0.5 \mathrm{~mL} / \mathrm{min}$ flow rate, and $10 \mu \mathrm{L}$ injection volume. A concentration of $1 \mathrm{mg} / \mathrm{mL}$ of each sample was used. The mobile phase consisted of $0.1 \%$ formic acid in water and $0.1 \%$ formic in acetonitrile. High-resolution mass spectrometry (HRMS) was carried out in both positive and negative ionization modes. The analysis had a run time of $25 \mathrm{~min}$ and a postrun time of $5 \mathrm{~min}$. Data generated from LC-MS were used in metabolomics analyses. A single technical replicate was used in this study. Furthermore, the antibacterial activities of the lichen and ELF fractions were also used (see Section 2.3).

\subsection{Metabolomics Profiling Studies}

LC-MS data were used for metabolomic profiling studies following the protocol of Macintyre et al. [33]. Raw data were first converted to mzML file format using the software ProteoWizard [34]. Data processing was carried out using the software MZMine 2 [35]. Key features of this process included detection (mass detector: centroid; noise level: 1000; minimum time span: $0.2 \mathrm{~min}$; minimum height: $1 \times 10^{4} ; \mathrm{m} / z$ tolerance: $0.001 \mathrm{~m} / \mathrm{z}$ or $5 \mathrm{ppm}$ ), deconvolution (chromatographic threshold: $5 \%$ ), deisotoping $(\mathrm{m} / \mathrm{z}$ tolerance: $0.001 \mathrm{~m} / \mathrm{z}$ or $5 \mathrm{ppm}$, retention time (RT) tolerance: 0.1 absolute $(\mathrm{min})$, maximum charge: 2 , representative isotope: most intense), filtering ( $\mathrm{m} / \mathrm{z}$ range: $100-1999, \mathrm{RT}: 0-45 \mathrm{~min})$, alignment $(\mathrm{m} / \mathrm{z}$ tolerance: $0.001 \mathrm{~m} / z$ or $5 \mathrm{ppm}, \mathrm{m} / z$ weight: 20 , RT tolerance: 5 relative $\%$ ) and gap filling ( $\mathrm{m} / \mathrm{z}$ tolerance: $0.001 \mathrm{~m} / z$ or $5 \mathrm{ppm}$, intensity tolerance: $1 \%$ ). Complete software settings were as described by Macintyre et al. [33]. A data clean-up was also carried out by creating an Excel macro that enabled positive and negative ionization data (exported as CSV file) to be processed together, minimizing the risk of missing poorly ionizing compounds only detected in one mode. The Excel macro was used to dereplicate the samples, matching each $m / z$ found in the samples with those in the Dictionary of Natural Products (DNP, version 2017) database to provide complete information of all the putative identified metabolites, as well as those that were unidentified. For the dereplication, a feature ID number, ionization mode, $m / z$, retention time, possible molecular formula, peak intensity, related compounds, and source of the specific metabolites (if available) were generated.

\subsection{Multivariate Analyses}

Multivariate analyses (MVAs) that included both Principal Component Analysis (PCA), Partial Least Squares-Discriminant Analysis (PLS-DA) and Orthogonal Projection to Latent Structures Discriminant Analysis (OPLS-DA) for the prediction of antibacterial metabolites were carried out using the software SIMCA ver. 15.0.2 (Umetrics, Umeå, Sweden) and MetaboAnalyst $\subseteq 4.0$ [32]. PCA was used to observe an overview of variance between the lichen and ELF crude extracts (predictor variables) and secondary metabolites (responses) generated from LC-MS data and to detect outliers that could influence the model. PLS-DA and OPLS-DA, on the other hand, were used to investigate the significant differences among metabolites produced by two different taxonomic origins (i.e., lichen and ELF) and their respective bioactivities (i.e., active and inactive samples against $S$. aureus), respectively. OPLS-DA was also used to identify the chemically distinct samples that may yield novel and bioactive secondary metabolites. A pareto scaling was applied to all MVA to reduce the influence of intense peaks while emphasizing weaker peaks that may have more biological relevance [36]. The $Q^{2}$ and $R^{2}$ values were reported as a qualitative measure of consistency between the predicted and original data. These values explained the goodness of prediction of the statistical models, representing the total explained variance and the predictive power of the models. The CV-ANOVA was also employed for further statistical validity of the models. The variable importance of projection or VIP scores were determined using the SIMCA software. This further validates the "ultimate" discriminating features defined on the PLS-DA plot, identifying the metabolites that greatly contribute to the variation of the groups. The greater the VIP score, the higher the discriminatory 
power of the metabolite. VIP scores $>1$ show the metabolites carrying highly discriminating information between classes [37].

\section{Results}

\subsection{Bioactivities of Lichen and ELF Erude Extracts}

Crude extracts of the lichen Usnea and their ELF were evaluated for antibacterial properties. These antibacterial data were separately reported and discussed in detail in our other studies $[14,15]$. In general, lichen crude extracts only showed inhibition against S. aureus, with $U$. bismolliuscula collected from the Philippines $(\mathrm{PH})$ showing the highest ZOI followed by $U$. baileyi $(\mathrm{PH})$. All lichen crude extracts gave a similar MIC value of $0.0625 \mathrm{mg} / \mathrm{mL}$ (Table 2). Meanwhile, six of the nine ELF crude extracts were active against $S$. aureus and E. coli, with Annulohypoxylon albidiscum and Xylaria venustula exhibiting the strongest activities against $S$. aureus, albeit having a high MIC value of $2.5 \mathrm{mg} / \mathrm{mL}$. Interestingly, $X$. venustula also exhibited activity against $E$. coli, with a MIC value of $5 \mathrm{mg} / \mathrm{mL}$ (Table 2).

Table 2. Minimum inhibitory concentration (MIC) and minimum bactericidal concentration (MBC) values of the nine ELF crude extracts against $S$. aureus and E. coli.

\begin{tabular}{|c|c|c|c|c|c|}
\hline ELF Species & Sample Code & Extract Yield ${ }^{1}(g)$ & $\begin{array}{l}\text { S. aureus ATCC } \\
25923(\mathrm{mg} / \mathrm{mL})\end{array}$ & $\begin{array}{l}\text { E. coli ATCC } 25922 \\
(\mathrm{mg} / \mathrm{mL})\end{array}$ & References $^{3}$ \\
\hline Xylaria sp. & Xsp & $3.873(0.48 \%)$ & MIC and MBC: 10 & MIC and MBC: 10 & \multirow{4}{*}{ Santiago et al. [15] } \\
\hline Fusarium solani & Fs & $3.196(0.40 \%)$ & MIC and MBC: 10 & No activity & \\
\hline Pseudopestalotiopsis theae & $\mathrm{Pt}$ & $1.4909(0.18 \%)$ & MIC and MBC: 10 & MIC and MBC: 10 & \\
\hline Xylariaceae sp. $^{2}$ & Xcsp & $2.357(0.29 \%)$ & MIC: 1.25; MBC: 2.5 & No activity & \\
\hline Astrocystis bambusae & $\mathrm{Ab}$ & $3.368(0.42 \%)$ & MIC: 10; MBC: >10 & No activity & \multirow{5}{*}{ Santiago et al. [14] } \\
\hline Annulohypoxylon albidiscum & $\mathrm{Aa}$ & $3.630(0.45 \%)$ & MIC: 2.5 ; MBC: 5 & No activity & \\
\hline Daldinia eschscholtzii & De & $2.755(0.34 \%)$ & MIC and MBC: 10 & No activity & \\
\hline Nemania bipapillata & $\mathrm{Nb}$ & $2.300(0.29 \%)$ & MIC and MBC: 10 & MIC and MBC: 10 & \\
\hline Xylaria venustula & $\mathrm{Xv}_{\mathrm{v}}$ & $9.767(1.22 \%)$ & MIC and MBC: 2.5 & MIC and MBC: 5 & \\
\hline
\end{tabular}

${ }^{1}$ Crude extracts prepared from $800 \mathrm{~g}(5 \times 160 \mathrm{~g})$ rice culture media. ${ }^{2}$ Discontinued due to unstable reproducibility to regrow the fungus during scale-up. ${ }^{3}$ References where the MIC and MBC values were reported earlier.

\subsection{Metabolomic Profiles of Lichen and ELF Crude Extracts}

The crude extract of $U$. baileyi (MY, UbyMY) yielded a higher metabolite density and diversity, with molecular weights ranging from 400 to 1400 Dalton (Da) as indicated by the heatmap (Figure 1). However, no further metabolite profiling was carried out on U. baileyi collected from the Philippines $(\mathrm{PH})$ due to very low yield of extract that was less than a milligram. U. pectinata samples (UpecMY and UpecPH), on the other hand, also revealed comparable densities and diversity of metabolites in the same molecular weight range. The molecular weights of the metabolites present in UpecPH ranged between 200 and $1400 \mathrm{Da}$ (Figure 1). However, the heatmap, along with the PCA scores plot (Figure S1), revealed that UpecPH and UpecMY gave different chemical profiles as they were situated farther from each other on the dendrogram (Figure 1) and their cluster sphere (Figure S1). Furthermore, crude extracts of $U$. bismolliuscula collected from MY (UbsMY) and PH (UbsPH) gave very similar and almost identical chemical profiles, as indicated by both heatmap-dendrogram and PCA scores plot, where both samples clustered together. Molecular weights of the metabolites detected in UbsMY and UbsPH ranged between 250 and $1400 \mathrm{Da}$ (Figure 1). The MVA model gave an $R^{2}$ value of 0.99 , a $Q^{2}$ value of 0.72 , and accuracy of 1 using Pareto scaling at two components. 


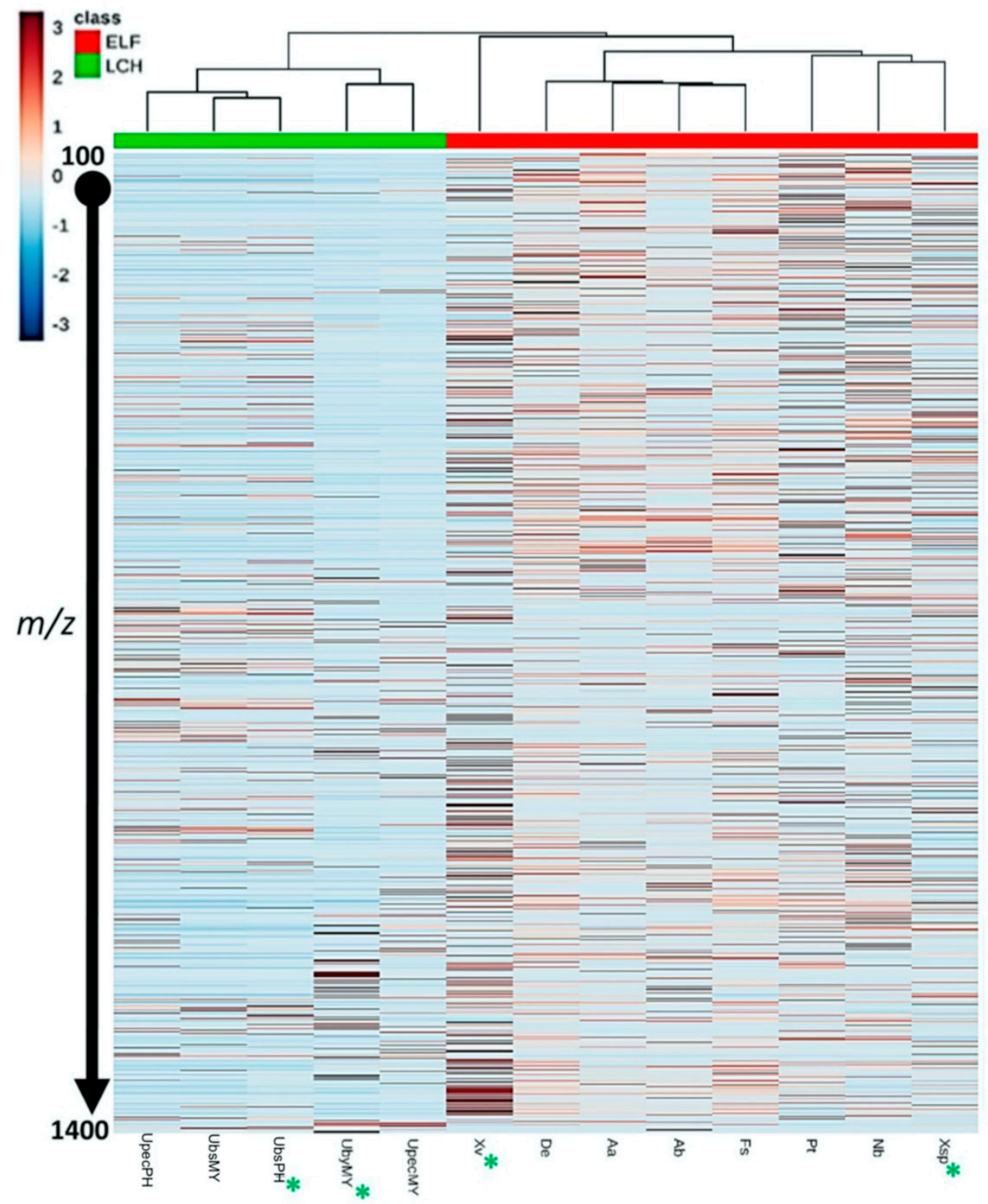

Figure 1. Heatmap and dendrogram of crude lichen and ELF extracts. The presence of more red bars indicates higher diversity in their chemical profile. Those marked with an asterisk $\left({ }^{*}\right)$ were chosen for further fractionation work (ELF: endolichenic fungi, LCH: lichen).

In general, ELF crude extracts indicated a higher diversity in their metabolomic profiles when compared to their lichen hosts. Among the ELF crude extracts, Xylaria venustula (PH, $\mathrm{Xv}_{\mathrm{v}}$ ) afforded the most diverse metabolomic profile as shown on the heatmap-dendrogram (Figure 1). This was followed by Pseudopestalotiopsis theae (MY, Pt), Nemania bipapillata $(\mathrm{PH}, \mathrm{Nb})$ and Xylaria sp. (MY, Xsp). Molecular weights of the metabolites found in these four ELF isolates ranged between 100 and $1400 \mathrm{Da}$. Interestingly, both Xsp and Xv were separated from the main cluster of other ELF samples as shown by the scores plot (Figure S1), indicating their unique chemical profiles.

\subsection{Bioactivities of Lichen and ELF Fractions}

In this study, the crude extracts of $U$. bismolliuscula (UbsMY and UbsPH), U. baileyi (UbyMY) and U. pectinata ( $\mathrm{PH}, \mathrm{Upec} \mathrm{PH})$ offered relatively diverse chemical profiles. In terms of their antibacterial activities, $\mathrm{UbsPH}$ exhibited the strongest activity against $\mathrm{S}$. au- 
reus (16 $\mathrm{mm} \mathrm{ZOI}$ ) among all the lichen crude extracts, followed by UbyMY (12 mm ZOI). Therefore, UbsPH and UbyMY were chosen for further fractionation work affording six and four fractions, respectively. From the UbsPH fractions, UbsFr5 was found to be active (10 mm ZOI), followed by UbsFr6 (7.7 mm ZOI), while the rest of the other tested fractions did not manifest any inhibition (Figure 2a). Interestingly, UbsFr5 exhibited higher inhibition than the reference usnic acid. From UbyMY, only UbyFr9 was found to be active against S. aureus ( $8.2 \mathrm{~mm} \mathrm{ZOI).}$

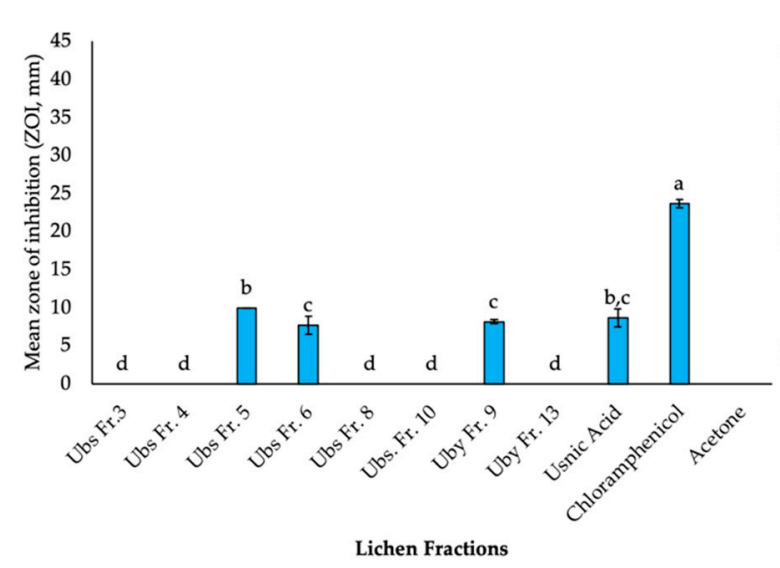

(a)

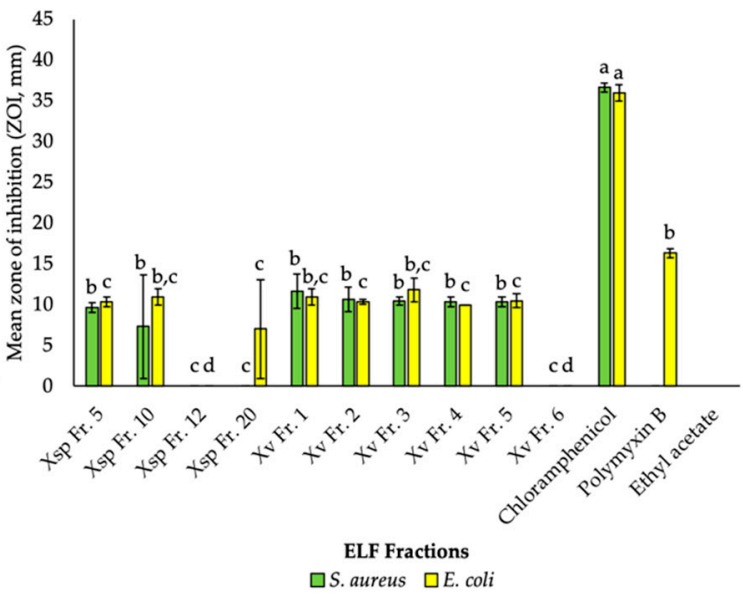

(b)

Figure 2. Biological activities of lichen and ELF fractions. (a) Antibacterial activities of U. bismolliuscula (PH, UbsPH) and U. baileyi (MY, UbyMY) fractions against S. aureus ATCC 25923. (b) Antibacterial activities of Xylaria sp. (MY, Xsp) and X. venustula $(\mathrm{PH}, \mathrm{Xv})$ fractions against S. aureus ATCC 25923 and E. coli ATCC 25922. Standard deviation values are indicated by the error bars $(p<0.05)$. Letters above error bars indicate the statistical significance within groups using one-way ANOVA and Tukey HSD. a, b, c, d different letters indicated on the columns denote statistical significance between respective fractions.

In this study, $X_{\mathrm{V}}$ and $\mathrm{Xsp}_{\mathrm{sp}}$ were the most abundant isolated ELF. $\mathrm{X}_{\mathrm{V}}$ also produced the highest extract yield of $9.8 \mathrm{~g}(1.22 \%)$, followed by Xsp with $3.9 \mathrm{~g}(0.48 \%)$ (Table 1$)$. For their bioactivities, $X_{\mathrm{v}}$ gave MIC and MBC values of 2.5 and $5 \mathrm{mg} / \mathrm{ml}$ against $S$. aureus and E. coli, respectively (Table 2). Xsp, on the other hand, only gave an MIC of $10 \mathrm{mg} / \mathrm{mL}$ against both test bacteria. There were also other ELF isolates, such as Xylariaceae sp. (Xcsp) and Annulohypoxylon albidiscum (Aa), that exhibited even stronger antibacterial activities than $X_{v}$ and $X_{s p}$. However, these isolates only exhibited activities against S. aureus, and hence, were not prioritized in this study. In addition, $\mathrm{Xcsp}$ was discontinued due to its poor growth reproducibility in the flask cultures. The chemical profile of Aa also afforded less diversity of compounds. Due to chemical diversity, dual activity against both test organisms, as well as good yield of extracts, further fractionation was achieved with Xsp and $X_{v}$ yielding seven and six fractions, respectively. From $X_{s p}$, only the fractions $X_{s p F r 1}$ and XspFr10 were found to be active against both S. aureus and E. coli (Figure 2b). Similarly, fractions $X_{v} F r 1$ to 5 from $X_{v}$ also showed significant activities against both test strains (Figure 2b). Low yielding fractions were not feasible for any of the assays used in this study.

\subsection{Chemical Profile Differences between Host Lichens and Associated ELF}

Mass spectral data of 36 lichen and ELF crude extracts and fractions were subjected to MVA. Usnic acid was used as a reference standard to validate the positioning of the Usnea samples in the various MVA plots. PCA of the lichen and ELF crude extracts and fractions gave a good separation between the host lichen and their associated fungi. However, in the PCA plots, all ELF samples clustered together except for the outlying samples originating from $X$. venustula $\left(\mathrm{Xv}_{\mathrm{v}}\right.$. The score scatter plot (Figure 3a) revealed two distinct fractions, $\mathrm{X}_{\mathrm{vFr}}$ and $\mathrm{X}_{\mathrm{vFr}}$. A heatmap (Figure S2) displays the comparative distribution of the 
metabolites ranked by t-tests in the respective classes and indicated the presence of a set of collective metabolites for the antibacterial extract $X_{v}$ along with its bioactive fractions $\mathrm{X}_{\mathrm{vFr}}, \mathrm{X}_{\mathrm{vFr}} 3$ and $\mathrm{XvFr}_{\mathrm{v}}$ (in yellow box, Figure S2). It was also observed that the Xylaria samples ( $X_{s p}$ and $X_{v}$ ) afforded a unique set of metabolites, which did not align with those of the other ELF samples as further shown on the heatmap (in blue box, Figure S2). The occurrence of usnic acid in Usnea extracts (UpecMY, UpecPH, UbsMY, and UbsPH) representing the lichens $U$. pectinata and $U$. bismolliuscula was also be revealed on the heatmap (Figure S2). However, usnic acid was not detected in U. baileyi (UbyMY).

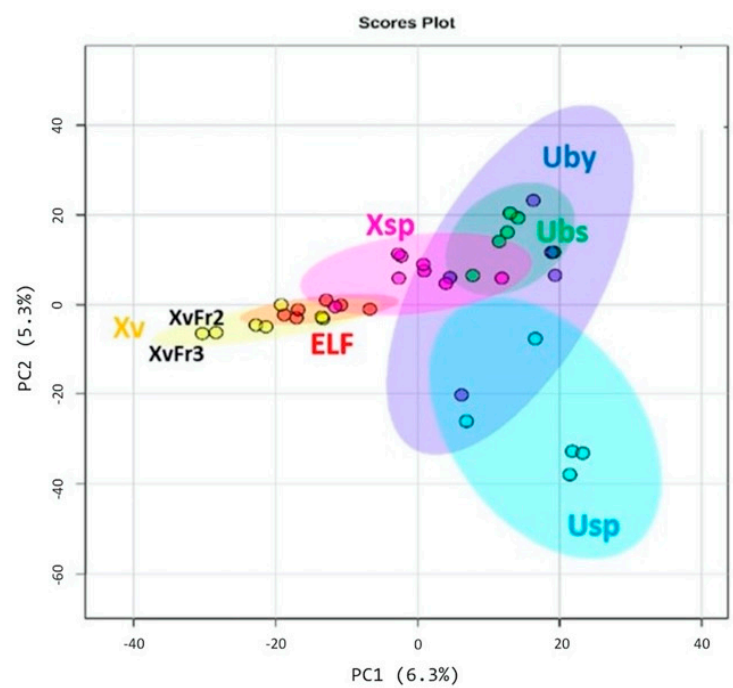

(a)

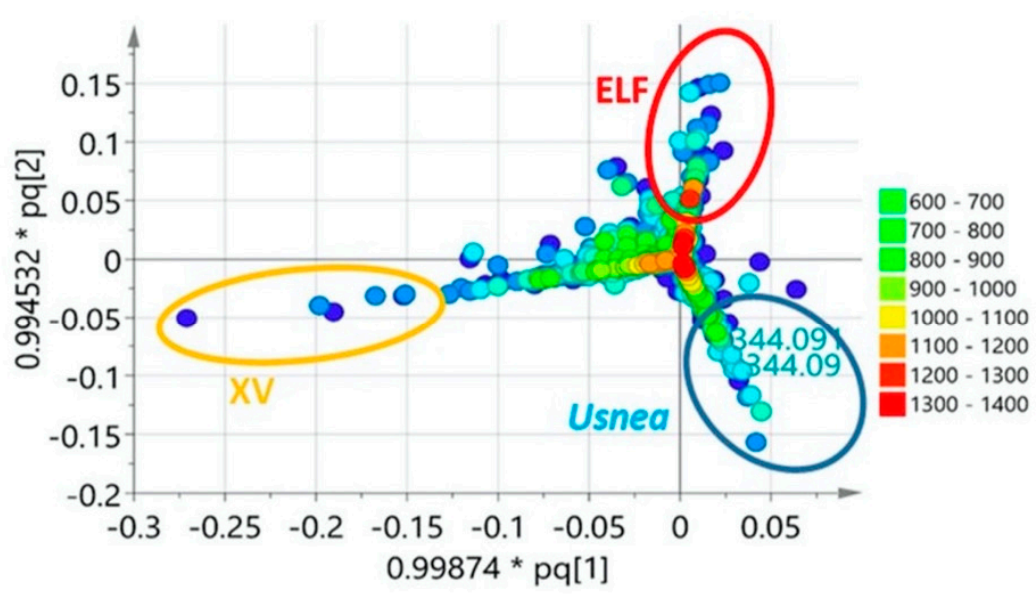

(b)

Figure 3. Multivariate analyses of the LC-MS data of 36 lichen and ELF crude extracts (including the reference (+)-usnic acid) and selected fractions. (a) principal component analysis (PCA) scores plot revealed distinct Xv fractions away from the overlapping clusters. (b) Encircled features on partial least squares discriminant analysis (PLS-DA) loading plot generated from SIMCA revealed metabolites (in molecular weight) responsible for the separation of the respective taxonomical groups. (ELF: endolichenic fungi, UBS: U. bismolliuscula, UBY: U. baileyi, USP: U. pectinata, XSP: Xylaria sp., XV: X. venustula). The pq vectors indicate $99.87 \%$ and $99.45 \%$ influence of individual $X$ - and Y-variables, respectively, to the model suggesting very good prediction of underlying patterns in the data.

A similar result was amplified by the PLS-DA loading plot generated from SIMCA (Figure $3 \mathrm{~b}$ ), showing clearly the discriminating metabolites that differentiated the Usnea host lichens from its associated ELF, as well as the separation of the Xylaria samples from the rest of the ELF strains investigated in this study. The occurrence of usnic acid with the molecular weight of 344.09 Da specified the clustering of the Usnea samples. The PLSDA loading plot (Figure $3 b$ ) disclosed unique discriminating metabolites with molecular weights of 100 to 300 Da representing the Xylaria groups, while the rest of the ELF samples covered molecular weights of 200 to $400 \mathrm{Da}$. However, their Usnea hosts yielded metabolites with higher molecular weights of 300 to $500 \mathrm{Da}$. The metabolites that separated the fractions of $X_{v}$ were the same as those that discriminated the distinct position of $X_{v} F r 2$ and $X_{v} F r 3$ on the PCA scores plot. The PLS-DA loading plot (Figure $3 \mathrm{~b}$ ) also displayed the molecular ion masses of the secondary metabolites responsible for discriminating $X_{v} F r 2$ and $X_{v} F r 3$.

In a PLS-DA scores plot (Figure 4), lichen and ELF crude extracts and fractions were grouped according to their taxonomical origins. The model achieved an $R^{2}$ value of 1 , a $Q^{2}$ value of 0.56 , and accuracy of 0.81 using Pareto scaling at five components. The PLS-DA score scatter plot afforded the separation of four distinct groups representing the extracts of the lichen hosts $U$. baileyi (Uby), $U$. bismolliuscula (Ubs), and $U$. pectinata (Usp) on one group, while their associated ELF along with their respective fractions were divided into three clusters (ELF, $X_{s p}$ and $\mathrm{Xv}$ ) (Figure 4). Xylaria sp. (Xsp) and X. venustula $\left(\mathrm{Xv}_{\mathrm{v}}\right)$ 
extracts together with their fractions were separated from the ELF cluster. SIMCA found a variation of $12.5 \%$ for the separation between groups while the intermodal variation was only $3.15 \%$, indicating a relatively good similarity in chemical profiles within respective clusters. A heatmap and dendrogram of lichen and ELF extracts and fractions (Figure S3a) based on the top 25 metabolites ranked according to their Variable Importance of Projection (VIP) scores were generated to validate the "ultimate" discriminating features from the PLS-DA loading plot (Figure $3 \mathrm{~b}$ ). These discriminating features demarcated the groupings of the samples into clusters shown on PLS-DA scores plot (Figure 4). The dominance of the $X_{v}$ metabolites was illustrated on the VIP score heatmap for the top 25 metabolites (Figure S3a), which again established the richness and unique chemical diversity that $X$. venustula offered. The top 15 metabolites were listed on a VIP score scatter plot indicating their relative abundance in their respective classes (Figure S3b).

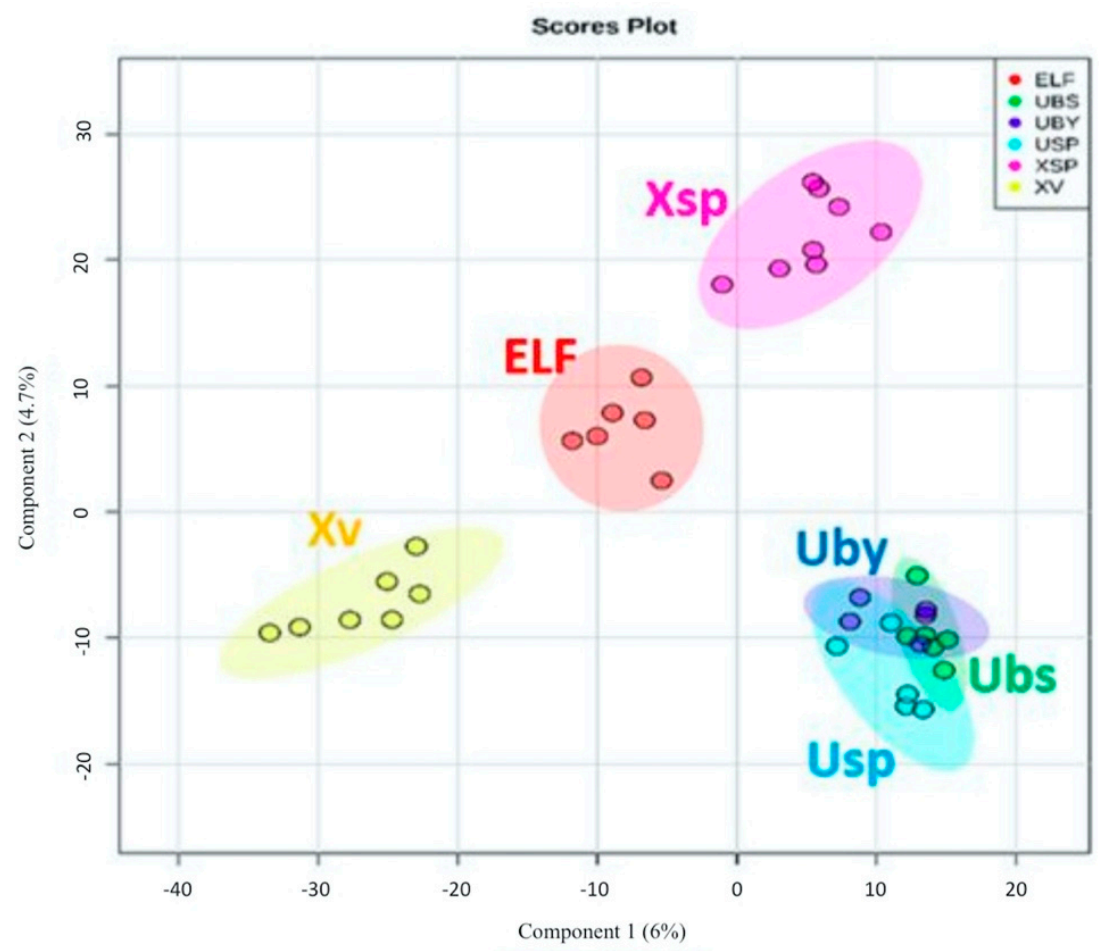

Figure 4. PLS-DA scores plot of lichen and ELF fractions grouped with their respective crude extracts and according to their taxonomical origin. (ELF: endolichenic fungi, UBS: $U$. bismolliuscula, UBY: $U$. baileyi, USP: U. pectinata, XSP: Xylaria sp., XV: X. venustula).

The discriminating features for the bioactive $\mathrm{Xv}$ extract and fractions were among those with VIP scores $>5$ that included $m / z$ (VIP scores) $m / z[\mathrm{M}+\mathrm{H}]^{+} 197.1173$ (9.83), $[\mathrm{M}+\mathrm{H}]^{+} 255.1233$ (6.65), $[\mathrm{M}-\mathrm{H}]^{-} 195.1031$ (6.54), and $[\mathrm{M}+\mathrm{H}]^{+} 273.1336$ (5.07), which were all primarily detected from the PLS-DA loading plot (Figure $3 b$ ) and dereplicated from the DNP database (Figure 5, Table 3). Ion peaks at $m / z[\mathrm{M}+\mathrm{H}]^{+} 197.117$ and 215.128 were identified to be the Xylaria metabolites and methyl xylariate $C$ and piliformic acid, respectively. The ion peaks at $m / z[\mathrm{M}-\mathrm{H}]^{-} 131.071$ and $[\mathrm{M}+\mathrm{H}]^{+} 273.134$ were dereplicated as the fungal natural products ophiocerin $\mathrm{B}$ and tensyuic acid C/D or pestalopyrone $\mathrm{A}$, respectively. The ion peaks at $m / z[\mathrm{M}+\mathrm{H}]^{+} 255.123$ and 274.274 were identified from the database as the sponge metabolites aplysinopsin and 2-amino-1,3-hexadecanediol or the marine-derived bacterial metabolite $\mathrm{N}$-dodecyl-diethanolamine, respectively. 
<smiles>CC(=O)C1=C(O)C2(Oc3cc(=O)c(C(C)=O)c(o3)-c3c(C(C)=O)c(C)c(O)c(O)c32)C(O)=C1C</smiles>

usnic acid<smiles>CCC/C=C(/C=C/C(=O)OC)C(C)=O</smiles>

methyl xylariate $\mathrm{C}$<smiles>CCCCC/C=C(/C(=O)O)[C@@H](C)C(=O)O</smiles>

piliformic acid<smiles>COC1=CC(=O)OC([C@@H](O)CCC(C)OC(C)=O)C1</smiles>

pestalopyrone $A$<smiles>C=C(C(=O)O)[C@H](CCCCCC(=O)OCC)C(=O)O</smiles>

tensyuic acid C<smiles>C=C(C(=O)O)[C@H](CCCCCC(=O)OC)C(=O)OC</smiles>

tensyuic acid D<smiles>C[C@H]1C[C@@H](O)[C@H](O)CO1</smiles>

ophiocerin B<smiles>CCCCCCCCCCCCC[C@H](O)[C@H](N)CO</smiles>

2-amino-1,3-hexadecanediol<smiles>CN1C(=N)N(C)C(=Cc2c[nH]c3ccccc23)C1=O</smiles>

aplysinopsin<smiles>CCCCCCCCCCCCN(CCO)CCO</smiles>

$\mathrm{N}$-dodecyl-diethanolamine

Figure 5. Structures of putatively dereplicated metabolites with usnic acid used as reference standard for lichen extracts in this study.

\subsection{Predicting the Bioactive Metabolites}

LC-MS data of the 37 samples were subjected to OPLS-DA (Figure 6) to predict the secondary metabolites responsible for their bioactivities. The samples were grouped into two classes: active vs. inactive (Figure 6a). The OPLS-DA score scatter plot gave an $\mathrm{R}^{2}$ value of 0.976 and a low $Q^{2}$ value of 0.0558 . The fractions $X_{v} F r 3$ and $X_{v F r} 4$ were revealed as the outliers while $\mathrm{XvFr}_{\mathrm{v}}$ just clustered towards the latter (Figure 6a). The model gave a good fit but very poor predictive power. This was due to the higher intermodal variation $\left(R^{2} X_{0}\right)$ of $21.7 \%$ while the variation between two groups $\left(R^{2} X\right)$ was only $3.57 \%$, indicating distinct differences in chemical profiles between the samples within the respective classes (Figure 6a). In parallel, the OPLS-DA loading plot (Figure 6b) displayed the secondary metabolites (P_60, N_592, P_1587, P_1117 and P_1588) assumed to be responsible for the bioactivity, as well as for discriminating the outliers (Table 3). These metabolites were among the discriminating features earlier defined in the previous section for the uniqueness of the $\mathrm{X}_{\mathrm{v}}$ extract and fractions. The additional ion peak at $\mathrm{m} / z 195.102$ eluting at $11.26 \mathrm{~min}$ is the negative ion $[\mathrm{M}-\mathrm{H}]^{-}$of $\mathrm{P} \_60$.

From the S-plot, the end features pinpointed the metabolites P_60, N_592, P_1587, P_1117, and P_1588 responsible for the antibacterial activity of $X$. venustula, which achieved $P$-values between 0.05 and 0.2 , with at least $80 \%$ confidence against the null hypothesis (Table 3). P_60 with a mass ion peak at $m / z[\mathrm{M}+\mathrm{H}]^{+} 197.117$ and a molecular weight of 196.11, was dereplicated as 4-acetyl-2,4-octanedienoic acid (methyl xylariate C) afforded a significant $P$-value of 0.05 . The exclusive occurrence of P_60, N_592, P_1587, P_1117, and P_1588 in the bioactive extract and fractions were further validated with box-and-whisker plots (Figure S4a) and their VIP scores were all >5 (Figure S4b). 
Table 3. Discriminating secondary metabolites for ion peaks of X. venustula MH370742 putatively dereplicated from Dictionary of Natural Products (DNP) database.

\begin{tabular}{|c|c|c|c|c|c|c|c|}
\hline MZMine ID $^{1}$ & $m / z$ & $\begin{array}{l}\text { Retention } \\
\text { Time }\end{array}$ & $\begin{array}{l}\text { Molecular } \\
\text { Weight }\end{array}$ & $\begin{array}{l}\text { Molecular } \\
\text { Formula }\end{array}$ & $\begin{array}{l}\text { Dereplicated } \\
\text { Identity }\end{array}$ & Reported Source $^{2}$ & $p$-Value \\
\hline P_60 & 197.117 & 11.55 & 196.11 & $\mathrm{C}_{11} \mathrm{H}_{16} \mathrm{O}_{3}$ & methyl xylariate $C$ & Xylaria NCY2 & 0.05 \\
\hline P_1117 & 215.128 & 11.41 & 214.121 & $\mathrm{C}_{11} \mathrm{H}_{18} \mathrm{O}_{4}$ & piliformic acid & $\begin{array}{l}\text { Poronia piliformis, } \\
\text { Xylaria longipes, X. } \\
\text { polymorpha, X. } \\
\text { hypoxylon, X. mali }\end{array}$ & 0.07 \\
\hline P_844 & 274.274 & 11.74 & 273.267 & $\mathrm{C}_{16} \mathrm{H}_{35} \mathrm{NO}_{2}$ & $\begin{array}{c}\text { 2-amino-1,3- } \\
\text { hexadecanediol } \\
N \text {-dodecyl- } \\
\text { diethabolamine } \\
\text { (DDE) }\end{array}$ & $\begin{array}{l}\text { Various sponges } \\
\text { marine-derived } \\
\text { bacteria }\end{array}$ & 0.09 \\
\hline N_592 & 195.103 & 13.29 & 196.11 & $\mathrm{C}_{11} \mathrm{H}_{16} \mathrm{O}_{3}$ & methyl xylariate $\mathrm{C}$ & Xylaria NCY2 & 0.11 \\
\hline P_1588 & 273.134 & 10.68 & 272.126 & $\mathrm{C}_{13} \mathrm{H}_{20} \mathrm{O}_{6}$ & $\begin{array}{c}\text { pestalopyrone } A \\
\text { tensyuic acid } C \\
\text { tensyuic acid D }\end{array}$ & $\begin{array}{l}\text { Pestalotiopsis } \\
\text { microspora } \\
\text { Aspergillus niger } \\
\text { FKI-2342 }\end{array}$ & 0.11 \\
\hline P_1587 & 255.123 & 10.83 & 254.116 & $\mathrm{C}_{14} \mathrm{H}_{14} \mathrm{~N}_{4} \mathrm{O}$ & aplysinopsin & $\begin{array}{l}\text { various marine } \\
\text { invertebrates }\end{array}$ & 0.11 \\
\hline N_215 & 131.071 & 7.25 & 132.079 & $\mathrm{C}_{6} \mathrm{H}_{12} \mathrm{O}_{3}$ & ophiocerin B & $\begin{array}{l}\text { Ophioceras } \\
\text { venezuelense }\end{array}$ & 0.18 \\
\hline
\end{tabular}

${ }^{1}$ MZMine ID includes ionization polarity: $\mathrm{P}$ - positive mode, $\mathrm{N}$-negative mode; ${ }^{2}$ reported source: organism(s) from where the secondary metabolites were isolated according to DNP database.

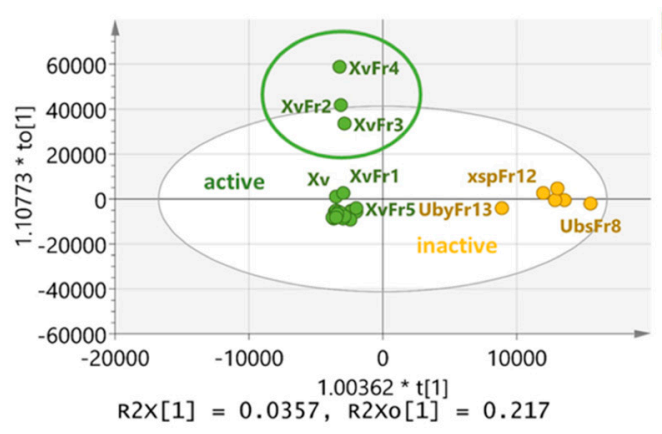

(a)

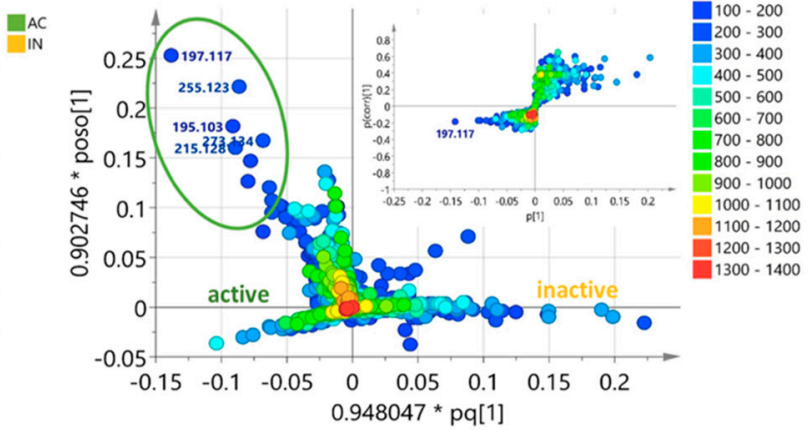

(b)

Figure 6. Multivariate analyses of 36 lichen and ELF crude extracts and selected fractions to predict the antibacterial metabolites in the Xylaria samples ( $\mathrm{Xsp}$ and $\mathrm{Xv}$ ). (a) Orthogonal projection to latent structures discriminant analysis (OPLS-DA) score scatter plot of samples categorized according to their bioactivities. (b) OPLS-DA loadings and S-plots with encircled features represent the bioactive metabolites of the most active samples. Active metabolites are on the left quadrants while inactive features are on the right quadrants. The pq and poso vectors indicate $94.80 \%$ and $90.27 \%$ influence of individual X-and Y-variables, respectively, to the model suggesting good prediction of underlying patterns in the data.

\section{Discussion}

In this study, the chemical profiles and antibacterial activities of different species of Usnea and their associated ELF strains were evaluated using metabolomics tools. Three species of Usnea collected from Malaysia and the Philippines-U. baileyi, U. bismolliuscula, and $U$. pectinata - were evaluated on their antibacterial activities. Consistent with previous studies $[29,38,39]$, results revealed that all lichen crude extracts were active against Gram-positive bacteria but inactive against Gram-negative bacteria (as reported in Santiago et al. [14,15]), albeit we only tested one representative bacterial species per category. Usnic acid, the major secondary metabolite found in the lichen Usnea, is known as the active substance responsible for uncoupling oxidative phosphorylation in bacteria, leading to cell death [40]. Other bioactive lichen metabolites such as norstictic and salazinic acids 
have also been reported in the genus Usnea [29]. In this study, however, only usnic acid was used as the reference lichen substance. A slight difference in the bioactivities in terms of the size of the ZOI was observed in this study between extracts of similar Usnea species collected from different sites. This could be due to quantitative differences in secondary metabolites produced by the respective lichens, which have consistently been described among lichen populations [41]. ELF crude extracts were found to be active against both the Gram-positive S. aureus and Gram-negative E. coli [14,15]. Although limited test organisms were evaluated in this study as we only wanted to initially explore their potential bioactivities, ELF were found as good sources of bioactive compounds and may offer medicinal and industrial advantages over their lichen hosts. Similar observations were reported by Padhi and Tayung [42].

In relation to the crude extracts, fractions obtained from lichen hosts and ELF that exhibited antibacterial activities were evaluated and compared. As expected, lichen fractions were only active against $S$. aureus, while few ELF fractions were active against both test bacteria. There were, however, fractions that did not exhibit any activities against the test bacteria, despite the strong antibacterial activity of its crude extract. This could be due to the absence of other features in the crude extract found earlier that behaved synergistically and, as a result of fractionation, the metabolites were separated from each other, hence resulting in the loss of bioactivity [43].

In this study, PCA, an unsupervised MVA method, was performed using LC-HRMS data. The score scatter plot gave an overview of the samples' chemical profiles, while determining the relationship between groups [36]. In this study, the generated PCA showed the groupings of samples based on their secondary metabolite profiles. $R^{2}$ at $c a .0 .99$, $\mathrm{Q}^{2}>0.72$, and an accuracy of 1 suggested good fit and predictive power of the model. The generated score scatter plot revealed very good separation between the lichen hosts and their associated ELF. The Xylaria samples, $X_{\mathrm{v}}$ and $\mathrm{Xsp}$, were distinctly situated farthest from the main ELF cluster, which suggests the uniqueness of their chemical profiles. Such an observation supports the hypothesis that ELF produce metabolites distinct from their lichen hosts [17].

PCA and PLS-DA of the fractions, along with their respective extracts, were also performed. In this study, unique secondary metabolites were identified from the ELF X. venustula. All of these metabolites, to the best of our knowledge, are yet to be reported for ELF. The discriminating features of the $X_{v}$ fractions were caused mainly by six secondary metabolites (P_60, P_844, P_1117, P_1587, P_1588, and N_215). The metabolites P_60 and P_1117 represented chemical uniqueness for the distinct $X_{v}$ fractions, thus prompting their separation from the rest of the ELF extracts and fractions. P_60 and P_1117 were putatively identified as methyl xylariate $C$ (or 4-acetyl-2,4-octadienoic acid) and piliformic acid, respectively. These were first isolated from an endophytic fungus Xylaria and reported to exhibit antimicrobial and cytotoxic activities [44,45]. As such, identifying these compounds from an endolichenic fungus Xylaria is feasible. In addition, it was previously reported that ELF produce secondary metabolites with carbon skeletons similar to those of the bioactive compounds produced by endophytes and are hypothesized to perform similar ecological roles for the host organism [17]. As such, similar gene clusters are expressed, resulting in the production of comparable metabolites by both endophytic and endolichenic fungi belonging to the same family - e.g., Xylariaceae. Furthermore, the similarity in their carbon skeletons despite being produced by different host organisms may also result in similar biological properties as observed in previous studies [45].

The metabolite P_1588 was dereplicated as pestalopyrone A and tensyuic acid C or D. The antifungal agent pestalopyrone A was first described from the endophytic fungus Pestalotiopsis microspora [46]. On the other hand, tensyuic acids (C and D) were previously isolated from the terrestrial fungus Aspergillus niger and were reported to have moderate antibacterial activities against Bacillus subtilis [47]. The tensyuic acid derivatives are acyclic congeners of pestalopyrone A. Interestingly, the structure of piliformic acid is also very closely related to tensyuic acid where the alkyl chain is attached on the exomethylene unit. 
Aplysinopsin (P_1587) is a tryptophan derivative that was first isolated from the marine sponge genus Thorecta [48], and has been isolated from other marine invertebrates [49]. This compound has been reported for its broad spectrum of bioactivities such as antimicrobial, antiparasitic, and cytotoxic activities [49]. Additionally, congeners of 2-amino-1,3-hexadecanediol ( $\mathrm{P} \_844$ ) have been isolated from marine-derived bacteria and other marine invertebrates [50]. Alternatively, P_844 was also dereplicated as $\mathrm{N}$-dodecyldiethanolamine (DDE) and has been described as displaying a broad spectrum of activity towards Escherichia coli, which may have been influenced by the formation of micelles [51]. These latter compounds were never reported in the literature as lichen or fungal metabolites from terrestrial sources, implying that these discriminating ion peaks could also belong to metabolites structurally different to the dereplicated compounds and could instead be plausibly rendered as new natural products.

In this study, it can be observed that most identified metabolites were previously reported from other organisms such as plants, soil fungi (pathogens and endophytes), and marine organisms. It was has been postulated that ELF is a precursor to major ecological transitions such as pathogenicity, symbiosis, and endophytism [11]. As such, isolation of metabolites previously reported from endophytic or pathogenic fungi may also be expected for ELF. Their metabolic pathways are alike; hence, production of similar secondary metabolites is feasible.

OPLS-DA was employed to predict the secondary metabolites responsible for the antibacterial activities of all samples by grouping them between active and inactive. The model's $\mathrm{R}^{2}$ was 0.976 , indicating a good fit. The results were interpreted and it was found that $24.2 \%$ of the $x$ variables could be used to describe $91.7 \%$ of the variation between active and inactive samples. The OPLS-DA score scatter plot revealed the clear separation of $X_{v F r 2}, X_{v F r} 3$, and $X_{v F r} 4$ from the rest of the bioactive fractions and extracts, which again suggests their chemical uniqueness. $\mathrm{XvFr}_{\mathrm{F}}$ gave the most potent antibacterial activities against $S$. aureus and E. coli. From the loadings, five discriminating secondary metabolites were displayed and were assumed to be responsible for the observations in the score scatter plot. Interestingly, these five secondary metabolites were the same set of metabolites revealed as distinct in the PCA model based on the chemical profiles of all investigated samples.

To further pinpoint the most significant metabolites responsible for the bioactivity, an S-plot was generated. In this study, the metabolite with the molecular weight of $196.11 \mathrm{Da}$ (P_60, $m / z$ 197.117) is situated on the tip of the "wing" of the active side of the S-plot far from the rest of the metabolites. This metabolite was dereplicated as 4-acetyl-2,4octadienoic acid or methyl xylariate $\mathrm{C}$. It has a high correlation to the model along with a high contribution to the variance making it the best prospective diagnostic metabolite for the bioactive Xylaria venustula samples. Through dereplication, this secondary metabolite was found to be common to Xylaria species. The ELF from which the respective metabolites were detected belongs to the same genus Xylaria, hence conducting further studies was not encouraged since this metabolite has already been explored.

Four secondary metabolites situated next to P_60 were observed. Except for ophiocerin B (N_215), three of the discriminating metabolites (aplysinopsin (P_1587), methyl xylariate C (N_592), and piliformic acid (P_1117)) have been described in the literature for their bioactivities. Ophiocerin B was isolated from the fungus Ophioceras venezuelense and was described as not exhibiting antifungal (against Candida albicans) and antibacterial (against S. aureus and E. coli) activities in standard disk assays at $200 \mu \mathrm{g} /$ disk [52]. Although ophiocerin B was found to be a distinct metabolite for the $\mathrm{Xv}$ fractions, it was not pinpointed among the bioactive metabolites. It should be noted, however, that the secondary metabolite N_592 is not identical to P_60 due to their different retention times. This may suggest that these two metabolites could be molecular isomers.

Visual interpretation of multivariate models, particularly the supervised models for OPLS-DA, could be potentially misleading unless accompanied by validating parameters [53]. In this study, the $\mathrm{R}^{2}$ value of the OPLS-DA model was close to 1 ; however, the 
$\mathrm{Q}^{2}$ value was low. This implies that the model explained the dataset well but has poor predictive power. Nevertheless, the CV-ANOVA $p$-values were identified. In this study, only methyl xylariate $\mathrm{C}\left(\mathrm{P} \_60\right)$ had a $p$-value of 0.05 , which is significant. The rest of the discriminating metabolites had $p$-values greater than 0.05 but less than 0.2 . It can be inferred that those secondary metabolites were detected by the model with approximately $15 \%$ of occurrence by chance. The $P$-values were at the margin of the significance level. However, the VIP scores of the pinpointed bioactivity were all greater than 5 . Therefore, these compounds can still be targeted as potential diagnostic metabolites for "dual" bioactivity against $S$. aureus and E. coli.

The role of ELF on the lichen symbiosis has still not been fully investigated and remains unknown, although some assumptions have been made. For instance, the occurrence of ELF is presumed to play an important ecological role in lichens by assisting in lichen formation and growth, as well as by protecting the lichen host against insect herbivores through the production of bioactive compounds [54]. Our findings offer another interesting insight on the possible contribution of ELF to their lichen hosts. In this study, it was observed that different microorganisms were targeted by the metabolites produced by the lichens and their associated ELF. The lichen Usnea only inhibited the growth of S. aureus, as also observed in other studies [29,55]. In contrast, ELF inhibited both S. aureus and E. coli. We also observed bigger ZOIs from the ELF culture extracts than the lichen extracts, albeit the MIC values were lower for the lichen extracts. Interestingly, the differences in the bioactivities of ELF and their host lichens were further supported by the discovery of different set of metabolites produced by these organisms, as revealed by our metabolomics analyses. As such, we have come to believe that ELF contribute to the lichen association by producing different metabolites that may target other "competitor" microorganisms. While ELF's motivation may be self-serving as they inhibit the growth of their potential competitors, their presence may have also protected the host lichens from other possible "harmful" or "invading" microorganisms. This has also been observed for fungal endophytes in plants, even though the plants and their associated fungal endophytes produce similar secondary metabolites [56]. Perhaps, by cultivating ELF in the presence of their host lichen thallus or lichen components, as those found in myco- and/or photobionts, may reveal similarities in metabolite production. These observations serve as an excellent stimulus to explore the metabolic pathways and metabolite production of both the lichen and its associated fungi.

\section{Conclusions}

Among the lichen and ELF samples evaluated in this study, the ELF X. venustula showed potentially novel and bioactive antibacterial metabolites against $S$. aureus. To our knowledge, this is the first application of metabolomics and dereplication on the lichen Usnea from Malaysia and the Philippines and their associated ELF. Through this technology, the chemical natures of all lichen and ELF samples were revealed, which led to the discovery of the distinctiveness of the secondary metabolites produced by these organisms. Such a discovery also showed an interesting insight into the "protective role" of ELF towards their lichen hosts. Furthermore, the statistical models presented in this study also assisted in the identification of significant bioactive metabolites from ELF, particularly aplysinopsin (P_1587), piliformic acid (P_1117), and pestalopyrone A/tensyuic acid C/tensyuic acid $\mathrm{D}$ (P_1588), for future isolation work. The metabolomics approach conducted in this study allowed the rapid identification and targeting of possible diagnostic metabolites for bioactive natural products.

Supplementary Materials: The following are available online at https: / www.mdpi.com/2079-7 737/10/3/191/s1, Figure S1: PCA score plot of crude lichen and ELF extracts; Figure S2: Heatmap showing the comparative distribution of the metabolites ranked by $\mathrm{t}$-tests in the respective classes (ELF: endolichenic fungi, UBS: U. bismolliuscula, UBY: U. baileyi, USP: U. pectinata, XSP: Xylaria sp., XV: X. venustula); Figure S3: (a) Heatmap and dendrogram of lichen and ELF extracts and fractions based on the top 25 metabolites (m/z-Rt), ranked according to their VIP scores. The most active 
fractions $(\mathrm{ZOI} \geq 10 \mathrm{~mm})$ are marked with an asterisk $\left.{ }^{*}\right)$. Red box: lichen extracts and fractions; blue box: ELF extracts and fractions. (b) VIP scores of top 15 metabolites. The boxes on the right indicate the relative abundance of the metabolite in the respective classes. (ELF: endolichenic fungi, UBS: U. bismolliuscula, UBY: U. baileyi, USP: U. pectinata, XSP: Xylaria sp., XV: X. venustula); Figure S4: Multivariate analyses of 36 lichen and ELF crude extracts and selected fractions to predict the antibacterial metabolites in the Xylaria samples (Xsp and $\left.X_{v}\right)$. (a) Box-and-whisker plots of the predicted bioactive metabolites $\left(m / z\right.$-Rt) indicating their relative occurrence in $X_{v}$ fractions. (b) VIP scores bar plot for top 15 discriminating metabolites from both active and inactive classes. Those marked with an asterisk $\left({ }^{*}\right)$ are from the active $X_{v}$ fractions, File S1: Supplementary Methods.

Author Contributions: Conceptualization, K.A.A.S., R.E.-E., Y.L.C., T.E.E.d.C. and A.S.Y.T.; methodology, K.A.A.S., Y.L.C. and R.E.-E.; software, R.E.-E.; validation, K.A.A.S. and R.E.-E.; formal analysis, K.A.A.S., R.E.-E., Y.L.C., T.E.E.d.C. and A.S.Y.T.; investigation, K.A.A.S., R.E.-E., T.E.E.d.C. and A.S.Y.T.; resources, R.E.-E. and A.S.Y.T.; data curation, K.A.A.S. and R.E.-E.; writing-original draft preparation, K.A.A.S.; writing-review and editing, K.A.A.S., R.E.-E., Y.L.C., T.E.E.d.C. and A.S.Y.T.; visualization, K.A.A.S., R.E.-E., Y.L.C., T.E.E.d.C. and A.S.Y.T.; supervision, R.E.-E., T.E.E.d.C. and A.S.Y.T.; project administration, K.A.A.S., R.E.-E., Y.L.C., T.E.E.d.C. and A.S.Y.T.; funding acquisition, K.A.A.S. and A.S.Y.T. All authors have read and agreed to the published version of the manuscript.

Funding: This research was funded by Tropical Medicine and Biology Multidisciplinary Platform (TMB) Seed Grant from Monash University Malaysia, grant number 5140921-318-00.

Institutional Review Board Statement: Not applicable.

Informed Consent Statement: Not applicable.

Data Availability Statement: The data presented in this study are available in supplementary materials here.

Acknowledgments: This research was fully supported by the Graduate Research Merit Scholarships from Monash University Malaysia.

Conflicts of Interest: The authors declare no conflict of interest.

\section{References}

1. Kosanić, M.; Ranković, B. Lichen secondary metabolites: Bioactive properties and pharmaceutical potential. Lichen Second. Metab. Bioact. Prop. Pharm. Potential 2015, 1-202. [CrossRef]

2. Calcott, M.J.; Ackerley, D.F.; Knight, A.; Keyzers, R.A.; Owen, J.G. Secondary metabolism in the lichen symbiosis. Chem. Soc. Rev. 2018, 47, 1730-1760. [CrossRef]

3. Shrestha, G.; Clair, L.L.S. Lichens: A promising source of antibiotic and anticancer drugs. Phytochem. Rev. 2013, 12, 229-244. [CrossRef]

4. Jha, B.N.; Shrestha, M.; Pandey, D.P.; Bhattarai, T.; Bhattarai, H.D.; Paudel, B. Investigation of antioxidant, antimicrobial and toxicity activities of lichens from high altitude regions of Nepal. BMC Complement. Altern. Med. 2017, 17, 1-8. [CrossRef]

5. Goel, M.; Sharmaa, P.K.; Dureja, P.; Rani, A.; Uniyal, P.L. Antifungal activity of extracts of the lichens Parmelia reticulata, Ramalina roesleri, Usnea longissima and stereocaulon himalayense. Arch. Phytopathol. Plant Prot. 2011, 44, 1300-1311. [CrossRef]

6. Vanga, N.R.; Kota, A.; Sistla, R.; Uppuluri, M. Synthesis and anti-inflammatory activity of novel triazole hybrids of (+)-usnic acid, the major dibenzofuran metabolite of the lichen Usnea longissima. Mol. Divers. 2017, 21, 273-282. [CrossRef]

7. Leal, A.; Rojas, J.L.; Valencia-Islas, N.A.; Castellanos, L. New $\beta$-orcinol depsides from Hypotrachyna caraccensis, a lichen from the páramo ecosystem and their free radical scavenging activity. Nat. Prod. Res. 2018, 32, 1375-1382. [CrossRef] [PubMed]

8. Vu, T.H.; Le Lamer, A.C.; Lalli, C.; Samson, J.B.M.; Dévéhat, F.L.-L.; Le Seyec, J. Depsides: Lichen metabolites active against hepatitis C virus. PLoS ONE 2015, 10, e0120405. [CrossRef]

9. Song, Y.; Dai, F.; Zhai, D.; Dong, Y.; Zhang, J.; Lu, B.; Luo, J.; Liu, M.; Yi, Z. Usnic acid inhibits breast tumor angiogenesis and growth by suppressing VEGFR2-mediated AKT and ERK1/2 signaling pathways. Angiogenesis 2012, 15, 421-432. [CrossRef] [PubMed]

10. Varol, M. Lichens as a Promising Source of Unique and Functional Small Molecules for Human Health and Well-Being, 1st ed.; Elsevier B.V.: Amsterdam, The Netherlands, 2018; Volume 60, ISBN 9780444641816.

11. Arnold, A.E.; Miadlikowska, J.; Higgins, K.L.; Sarvate, S.D.; Gugger, P.; Way, A.; Hofstetter, V.; Kauff, F.; Lutzoni, F. A phylogenetic estimation of trophic transition networks for ascomycetous Fungi: Are lichens cradles of symbiotrophic Fungal diversification? Syst. Biol. 2009, 58, 283-297. [CrossRef] [PubMed]

12. U'Ren, J.M.; Lutzoni, F.; Miadlikowska, J.; Arnold, A.E. Community Analysis Reveals Close Affinities Between Endophytic and Endolichenic Fungi in Mosses and Lichens. Microb. Ecol. 2010, 60, 340-353. [CrossRef] [PubMed]

13. Petrini, O.; Hake, U.; Dreyfuss, M.M. An Analysis of Fungal Communities Isolated from Fruticose Lichens. Mycologia 1990, 82, 444-451. [CrossRef] 
14. Santiago, K.A.A.; dela Cruz, T.E.E.; Ting, A.S.Y. Diversity and bioactivity of endolichenic fungi in Usnea lichens from the Philippines. Czech Mycol. 2020, 73, 1-19. [CrossRef]

15. Santiago, K.A.A.; dela Cruz, T.E.E.; Ting, A.S.Y. Diversity and bioactivities of culturable endolichenic fungi from three Usnea spp.: A case study in Bukit Larut, Malaysia. Saudi J. Biol. Sci. 2020. under review.

16. Tripathi, M.; Joshi, Y. Endolichenic Fungi: Present and Future Trends. In Endolichenic Fungi: Present and Future Trends; Springer: Berlin, Germany, 2019; pp. 27-47. ISBN 9789811372681.

17. Singh, B.N.; Upreti, D.K.; Gupta, V.K.; Dai, X.F.; Jiang, Y. Endolichenic Fungi: A Hidden Reservoir of Next Generation Biopharmaceuticals. Trends Biotechnol. 2017, 35, 808-813. [CrossRef]

18. Paranagama, P.A.; Wijeratne, E.M.K.; Burns, A.M.; Marron, M.T.; Gunatilaka, M.K.; Arnold, A.E.; Gunatilaka, A.A.L. Heptaketides from Corynespora sp. inhabiting the cavern beard lichen, Usnea cavernosa: First report of metabolites of an endolichenic fungus. J. Nat. Prod. 2007, 70, 1700-1705. [CrossRef] [PubMed]

19. He, J.W.; Chen, G.D.; Gao, H.; Yang, F.; Li, X.X.; Peng, T.; Guo, L.D.; Yao, X.S. Heptaketides with antiviral activity from three endolichenic fungal strains Nigrospora sp., Alternaria sp. and Phialophora sp. Fitoterapia 2012, 83, 1087-1091. [CrossRef]

20. Wang, Q.X.; Bao, L.; Yang, X.L.; Liu, D.L.; Guo, H.; Dai, H.Q.; Song, F.H.; Zhang, L.X.; Guo, L.D.; Li, S.J.; et al. Ophiobolins P-T, five new cytotoxic and antibacterial sesterterpenes from the endolichenic fungus Ulocladium sp. Fitoterapia 2013, 90, $220-227$. [CrossRef] [PubMed]

21. Samanthi, K.A.U.; Wickramarachchi, S.; Wijeratne, E.M.K.; Paranagama, P.A. Two new bioactive polyketides from Curvularia trifolii, an endolichenic fungus isolated from Usnea sp., in Sri Lanka. J. Natl. Sci. Found. Sri Lanka 2015, 43, 217-224. [CrossRef]

22. Xie, F.; Chang, W.; Zhang, M.; Li, Y.; Li, W.; Shi, H.; Zheng, S.; Lou, H. Quinone derivatives isolated from the endolichenic fungus Phialocephala fortinii are Mdr1 modulators that combat azole resistance in Candida albicans. Sci. Rep. 2016, 6, 1-10. [CrossRef]

23. Padhi, S.; Das, D.; Panja, S.; Tayung, K. Molecular Characterization and Antimicrobial Activity of an Endolichenic Fungus, Aspergillus sp. Isolated from Parmelia caperata of Similipal Biosphere Reserve, India. Interdiscip. Sci. Comput. Life Sci. 2016, 9 , 237-246. [CrossRef]

24. Covington, B.C.; McLean, J.A.; Bachmann, B.O. Comparative mass spectrometry-based metabolomics strategies for the investigation of microbial secondary metabolites. Nat. Prod. Rep. 2017, 34, 6-24. [CrossRef]

25. Nagana Gowda, G.; Raftery, D. Biomarker Discovery and Translation in Metabolomics. Curr. Metabolomics 2013, 1, 227-240. [CrossRef]

26. Ito, T.; Masubuchi, M. Dereplication of microbial extracts and related analytical technologies. J. Antibiot. 2014, 67, 353-360. [CrossRef]

27. Hubert, J.; Nuzillard, J.M.; Renault, J.H. Dereplication strategies in natural product research: How many tools and methodologies behind the same concept? Phytochem. Rev. 2017, 16, 55-95. [CrossRef]

28. Jantan, I.; Ahmad, A.S. Oleoresins of three Pinus species from Malaysian pine plantations. ASEAN Rev. Biodivers. Environ. Conserv. 1999, 1-9. [CrossRef]

29. Santiago, K.A.A.; Sangvichien, E.; Boonpragob, K.; dela Cruz, T.E.E. Secondary metabolic profiling and antibacterial activities of different species of Usnea collected in Northern Philippines. Mycosphere 2013, 4, 267-280. [CrossRef]

30. Wu, W.; Dai, H.; Bao, L.; Ren, B.; Lu, J.; Luo, Y.; Guo, L.; Zhang, L.; Liu, H. Isolation and structural elucidation of proline-containing cyclopentapeptides from an endolichenic Xylaria sp. J. Nat. Prod. 2011, 74, 1303-1308. [CrossRef]

31. CLSI. Performance Standards for Antimicrobial Susceptibility Testing; Twenty-Second Informational Supplement; Clinical and Laboratory Standards Institute: Pennsylvania, PA, USA, 2012; Volume 32, ISBN 1562387855.

32. Chong, J.; Wishart, D.S.; Xia, J. Using MetaboAnalyst 4.0 for Comprehensive and Integrative Metabolomics Data Analysis. Curr. Protoc. Bioinforma. 2019, 68, 1-128. [CrossRef] [PubMed]

33. Macintyre, L.; Zhang, T.; Viegelmann, C.; Martinez, I.J.; Cheng, C.; Dowdells, C.; Abdelmohsen, U.R.; Gernert, C.; Hentschel, U.; Edrada-Ebel, R.A. Metabolomic tools for secondary metabolite discovery from marine microbial symbionts. Mar. Drugs 2014, 12, 3416-3448. [CrossRef] [PubMed]

34. Kessner, D.; Chambers, M.; Burke, R.; Agus, D.; Mallick, P. ProteoWizard: Open source software for rapid proteomics tools development. Bioinformatics 2008, 24, 2534-2536. [CrossRef]

35. Pluskal, T.; Castillo, S.; Villar-Briones, A.; Orešič, M. MZmine 2: Modular framework for processing, visualizing, and analyzing mass spectrometry-based molecular profile data. BMC Bioinform. 2010, 11. [CrossRef]

36. Worley, B.; Powers, R. Multivariate Analysis in Metabolomics. Curr. Metab. 2013. [CrossRef]

37. Davis, V.W.; Schiller, D.E.; Eurich, D.; Sawyer, M.B. Urinary metabolomic signature of esophageal cancer and Barrett's esophagus. World J. Surg. Oncol. 2012, 10, 1-12. [CrossRef]

38. Maciag-Dorszyńska, M.; Wegrzyn, G.; Guzow-Krzemińska, B. Antibacterial activity of lichen secondary metabolite usnic acid is primarily caused by inhibition of RNA and DNA synthesis. FEMS Microbiol. Lett. 2014, 353, 57-62. [CrossRef]

39. Santiago, K.A.A.; Borricano, J.N.C.; Canal, J.N.; Marcelo, D.M.A.; Perez, M.C.P.; dela Cruz, T.E.E. Antibacterial activities of fruticose lichens collected from selected sites in Luzon Island, Philippines. Philipp. Sci. Lett. 2010, 3, 18-29.

40. Guo, L.; Shi, Q.; Fang, J.-L.; Mei, N.; Ali, A.A.; Lewis, S.M.; Leakey, J.E.A.; Frankos, V.H. Review of usnic acid and Usnea barbata toxicity. J. Environ. Sci. Heal. Part C 2008, 26, 317-338. [CrossRef] [PubMed]

41. Fahselt, D. Individuals and populations of lichens. In Lichen Biology; Nash, T., III, Ed.; Cambridge University Press: Cambridge, UK, 2008; pp. 252-273. 
42. Padhi, S.; Tayung, K. In vitro antimicrobial potentials of endolichenic fungi isolated from thalli of Parmelia lichen against some human pathogens. Beni-Suef Univ. J. Basic Appl. Sci. 2015, 4, 299-306. [CrossRef]

43. Nwodo, U.U.; Ngene, A.A.; Iroegbu, C.U.; Obiiyeke, G. Effects of fractionation on antibacterial activity of crude extracts of Tamarindus indica. African J. Biotechnol. 2010, 9, 7108-7113. [CrossRef]

44. Hu, Z.; Li, Y.; Lu, C.; Lin, T.; Hu, P.; Shen, Y. Seven Novel Linear Polyketides from Xylaria sp. NCY2 jackii Chun, collected from Jiangshi Nature Reserve Zone of Fujian Province, China, during November of 2004. This strain was determined to be Xylaria sp. based on its complete ITS1-5. 8S-ITS2 gen. Helv. Chim. Acta 2010, 93, 925-933. [CrossRef]

45. Elias, L.M.; Fortkamp, D.; Sartori, S.B.; Ferreira, M.C.; Gomes, L.H.; Azevedo, J.L.; Montoya, Q.V.; Rodrigues, A.; Ferreira, A.G.; Lira, S.P. The potential of compounds isolated from Xylaria spp. as antifungal agents against anthracnose. Brazilian J. Microbiol. 2018, 49, 840-847. [CrossRef] [PubMed]

46. Lee, J.C.; Yang, X.; Schwartz, M.; Strobel, G.; Clardy, J. The relationship between an endangered North American tree and an endophytic fungus. Chem. Biol. 1995, 2, 721-727. [CrossRef]

47. Hasegawa, Y.; Fukuda, T.; Hagimori, K.; Tomoda, H.; Omura, S. Tensyuic acids, new antibiotics produced by Aspergillus niger FKI-2342. Chem. Pharm. Bull. 2007, 55, 1338-1341. [CrossRef]

48. Kazlauskas, R.; Murphy, P.T.; Quinn, R.J.; Wells, R.J. Aplysinopsin, a new tryptophan derivative from a sponge. Tetrahedron Lett. 1977, 18, 61-64. [CrossRef]

49. Bialonska, D.; Zjawiony, J.K. Aplysinopsins-Marine indole alkaloids: Chemistry, bioactivity and ecological significance. Mar. Drugs 2009, 7, 166-183. [CrossRef]

50. Zhang, W.; Guo, Y.-W.; Gu, Y. Secondary Metabolites from the South China Sea Invertebrates: Chemistry and Biological Activity. Curr. Med. Chem. 2006, 13, 2041-2090. [CrossRef]

51. Lambert, P.A.; Smith, A.R.W. The mode of action of N (n Dodecyl)diethanolamine with particular reference to the effect of protonation on uptake by Escherichia coli. J. Gen. Microbiol. 1977, 103, 367-374. [CrossRef] [PubMed]

52. Reátegui, R.F.; Gloer, J.B.; Campbell, J.; Shearer, C.A. Ophiocerins A-D and ophioceric acid: Tetrahydropyran derivatives and an africane sesquiterpenoid from the freshwater aquatic fungus Ophioceras venezuelense. J. Nat. Prod. 2005, 68, 701-705. [CrossRef] [PubMed]

53. Wheelock, Å.M.; Wheelock, C.E. Trials and tribulations of omics data analysis: Assessing quality of SIMCA-based multivariate models using examples from pulmonary medicine. Mol. Biosyst. 2013, 9, 2589-2596. [CrossRef]

54. Yu, N.H.; Park, S.-Y.; Kim, J.A.; Park, C.-H.; Jeong, M.-H.; Oh, S.-O.; Hong, S.G.; Talavera, M.; Divakar, P.K.; Hur, J.S. Endophytic and endolichenic fungal diversity in maritime Antarctica based on cultured material and their evolutionary position among Dikarya. Fungal Syst. Evol. 2018, 2, 263-272. [CrossRef]

55. Dandapat, M.; Paul, S. Secondary metabolites from lichen Usnea longissima and its pharmacological relevance. Pharmacognosy Res. 2019, 11, 103-109. [CrossRef]

56. Ludwig-Müller, J. Plants and endophytes: Equal partners in secondary metabolite production? Biotechnol. Lett. 2015, 37, 1325-1334. [CrossRef] [PubMed] 